\title{
Efficacy of Yttrium-90 Time-of-Flight PET/CT in Post Radioembolization Imaging of Microsphere Biodistribution in Radiosynovectomy
}

\author{
Masoomi $\mathrm{M}^{1}$, Alnafisi $\mathrm{N}^{2}$, Alenezi $\mathrm{S}^{3}$, Aldousari $\mathrm{HA}^{4}$ and \\ Alshammari I ${ }^{1}$ \\ ${ }^{1}$ Department of Nuclear Medicine, Adan Hospital, MOH, Kuwait \\ 2Department of Nuclear Medicien, Mubarak Al-Kabeer Hospital, MOH, Kuwait \\ ${ }^{3}$ Department of Nuclear Medicine, Faculty of Medicine, University of KW, Kuwait \\ ${ }^{4}$ Jaber Al Ahmed Nuclear Medicine and Molecular Imaging Center, Kuwait
}

*Corresponding author: Michael Masoomi, Department of Nuclear Medicine and Molecular Imaging, Adan Hospital, MOH, Kuwait, Tel: (+)965 51250687; Email: masoomim@sky.com

\section{Abstract}

Introduction: ${ }^{90} \mathrm{Y}$ bremsstrahlung scintigraphy suffers from a low spatial resolution due to indirect imaging of a continuous scatter radiation spectrum without a pronounced photo-peak resulting in a coarse representation of the microsphere biodistribution. The authors are investigating the feasibility of ${ }^{90} \mathrm{Y}$ PET/CT TOF imaging as a technique to improve detection accuracy of ${ }^{90} \mathrm{Y}$ distribution and leakage post radiation synovectomy in relation to conclusive information of activity presence in the target area.

Procedures: A knee phantom with the compartments that is mimicking the bone and soft tissue scattering - attenuation was developed using saline solution and Dipotassium Hydrogen Phosphate (K2HP04). A dose of 185 MBq of 90Y confined to a small container was embedded in the knee phantom. A LYSO PET with a 64 slice CT, as well as a 16 slice SPECT/CT camera was used. PET scans were acquired using positron fraction $3.186 \times 10-5$, half-life 64.1 h, 15 min per bed position. For bremsstrahlung SPECT imaging the energy windows were set to $55-285 \mathrm{keV}$.

Results: An algorithm which incorporates TOF+PSF, non-TOF and 3D-OSEM were used for image acquisition and reconstruction. The images were assessed for spatial resolution, technical success and detection of non-target activity. The PET images appeared to be suffering from low true coincident rate. Qualitative evaluation showed superior spatial resolution of PET/CT over bremsstrahlung SPECT with a clear edge definition of the phantom. We are planning to apply the procedure on patient imaging, and it appears the ${ }^{90} \mathrm{Y}$ PET scans of patients for microsphere bio-distribution in radiosynovectomy is the first time to be done in Kuwait. 


\section{International Journal of Nuclear Medicine \& Radioactive Substances}

Conclusion: The preliminary study suggests, although ${ }^{90} \mathrm{Y}$ PET imaging is suffering from low true counts, but it has qualitative superiority and results are indicating that it can serve in two primary clinical roles in radiosynovectomy: (1) evaluating technical success (2) predicting treatment efficacy.

Keywords: Yittrium-90 (90Y); Radiosynovectomy; Time of Flight PET/CT; SPECT

\section{Introduction}

Yttrium-90 $\left({ }^{90} \mathrm{Y}\right)$ is a pure beta emitter with Maximum energy of $2.27 \mathrm{MeV}$ and Mean energy of $0.935 \mathrm{MeV}$. It has average soft-tissue range of $3.6 \mathrm{~mm}$ and the physical halflife of 2.7 days.

While ${ }^{90} \mathrm{Y}$ is traditionally considered to be a pure $\beta$ emitter, there is also a minor decay branch, resulting in the e+e- creation [1] which can facilitate PET imaging. Despite relatively low administered ${ }^{90} \mathrm{Y}$ activity and delayed imaging at $24 \mathrm{~h}$, high resolution PET images in a pattern concordant with bremsstrahlung imaging can be obtained. PET/CT imaging following ${ }^{90} \mathrm{Y}$ radiation synovectomy is feasible and would be useful in further delineating the location of the activity seen on bremsstrahlung imaging. This technique may also help to predict treatment response or potential local complications.

${ }^{90} \mathrm{Y}$ bremsstrahlung scintigraphy suffers from a low spatial resolution, ranging between 11 to $15 \mathrm{~mm}$ depending on the choice of energy window, collimator, and image processing [2-4] (Figure1).
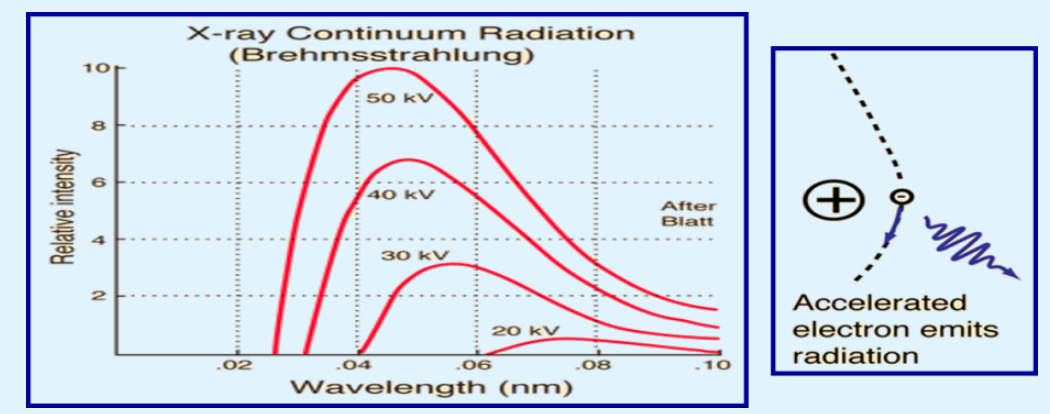

Figure 1: Bremsstrahlung X-ray.

This is due to its inherent technical limitation as a form of indirect imaging of a continuous scatter radiation spectrum without a pronounced photo-peak, resulting in a coarse representation of the microsphere bio distribution. ${ }^{90} \mathrm{Y}$ bremsstrahlung activity on SPECT/CT often appears smooth and diffuse, with ill-defined margins, which are difficult to distinguish from closely adjacent activity foci. As a result, assessment of activity within sub centimeter tumors or tumor vascular thrombosis are often suboptimal on ${ }^{90} \mathrm{Y}$ bremsstrahlung SPECT/CT. Quantitatively, ${ }^{90} \mathrm{Y}$ bremsstrahlung scintigraphy is largely inaccurate, despite compensation techniques for attenuation, scatter, and collimatordetector response, making it unsuitable for doseresponse analysis [5-7].

Positron emission tomography with integrated computed tomography (PET/CT) represents a technological leap from ${ }^{90} \mathrm{Y}$ bremsstrahlung SPECT/CT, both qualitatively and quantitatively, allowing direct imaging of ${ }^{90} \mathrm{Y}$ microspheres. Although ${ }^{90} \mathrm{Y}$ is predominantly a $\beta$-emitter, coincidence imaging of ${ }^{90} \mathrm{Y}$ is possible because of a minor decay branch to the $0+$ first excited state of zirconium ${ }^{90}$ followed by $\beta-\beta+$ internal pair production at a very low branching ratio of $31.86 \pm$ $0.47 \times 10-6$ [8-11]. However, the clinical application of ${ }^{90} \mathrm{Y}$ PET/CT has been fraught with challenges.

Today's time of flight PET/CT scanners uses lutetium based crystals due to its superior timing resolution. Such crystals, such as lutetium- yttrium- oxyortho silicate (LYSO), contain $2.6 \%$ of naturally occurring lutetium 176 (176Lu) which results in a background activity of intrinsic true and random coincidences which cannot be distinguished from coincidences by an external radioactive source [12-15]. This issue was previously 
thought to be clinically irrelevant until recent interest in ${ }^{90} \mathrm{Y}$ PET/CT. In addition, the low positron activity of ${ }^{90} \mathrm{Y}$ and the intrinsic activity of ${ }^{176} \mathrm{Lu}$ can have an impact on scatter correction. For time of flight PET, several authors have computed the scatter based on the PET activity [1618]. Since the scatter correction algorithm depends on the PET activity map, which is poor due to low signal and intrinsic noise, scatter correction will be negatively affected. The unfavorable combination of intrinsic background activity and a very low positron fraction result in reconstructed ${ }^{90} \mathrm{Y}$ PET/CT images which at the outset, seem un-interpretable due to high background noise.

Despite this, several institutions have published encouraging early experiences using ${ }^{00} \mathrm{Y}$ time of flight PET for post radioembolization imaging [19-21]. These early reports suggest that despite background noise, it is possible to obtain high resolution images of microsphere biodistribution using conventional time of flight PET scanners, with only minor adjustments to scan technique and image reconstruction such as PET acquisition time, number of bed positions, reconstruction iterations, and subsets.

From a clinical standpoint, ${ }^{90} \mathrm{Y}$ PET provides a superior post infusion evaluation of treatment technical success owing to its improved resolution. Absorbed dose maps generated from quantitative PET data can be used to predict treatment efficacy and manage patient follow up. For patients who receive multiple treatments, this information can also be used to provide patient specific treatment planning for successive therapies, potentially improving response. The broad utilization of ${ }^{90} \mathrm{Y}$ PET-CT has the potential to provide a wealth of dose-response information, which may lead to the development of improved radioembolization treatment planning models in the future. Furthermore, after radiation synovectomy and microsphere treatment a delayed PET-CT acquisition at $24 \mathrm{~h}$ can assist to investigate potential extra- articular radiotracer extravasation.

The investigation was a pilot methodology study attempting to investigate feasibility of ${ }^{90} \mathrm{Y}$ PET/CT imaging as a proposed technique, to improve overall detection accuracy of ${ }^{90} \mathrm{Y}$ distribution and leakage post radiation synovectomy. Furthermore, to compare the ability of ${ }^{90} \mathrm{Y}$ PET/CT versu ${ }^{90} \mathrm{Y}$ bremsstrahlung SPECT/CT in providing conclusive information about the presence or absence of ${ }^{90} \mathrm{Y}$ activity within the target area.

\section{Materials and Methods}

The radiosynovectomy technique involves insertion of an 18-gauge needle through the medial aspect of the knee positioned at 45 degrees flexion, aspiration of joint fluid, followed by injection of yttrium-90 citrate. Corticosteroid is next injected and flushed with saline. The knee is then tightly bandaged (Figure 2).

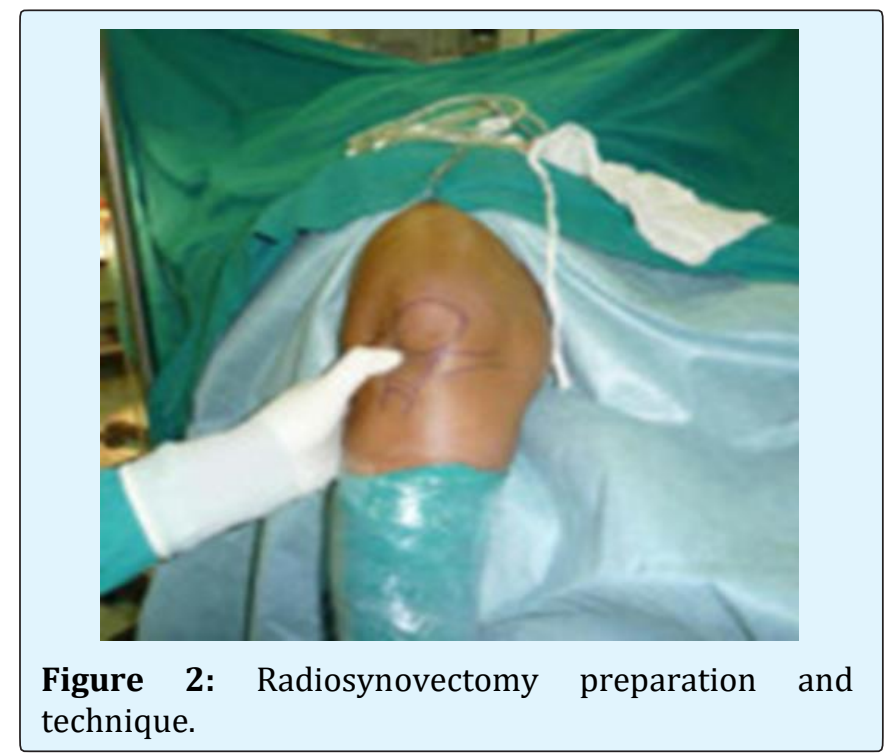

At the initial stage of our study a small neck phantom with the composition equivalent to soft tissue were utilised and a vial containing $185 \mathrm{MBq}^{90} \mathrm{YCl}_{3}$ (the same amount as a patient dose) was embedded inside the phantom to be imaged. At the later stage an in-house phantom to imitate the knee region with the compartments that is mimicking the bone and soft tissue scattering - attenuation was developed using saline solution (with the atomic number close to soft tissue) and Dipotassium Hydrogen Phosphate (K2HPO4) that has the atomic number $(\mathrm{Z})$ close to bone (Figure 3 ).

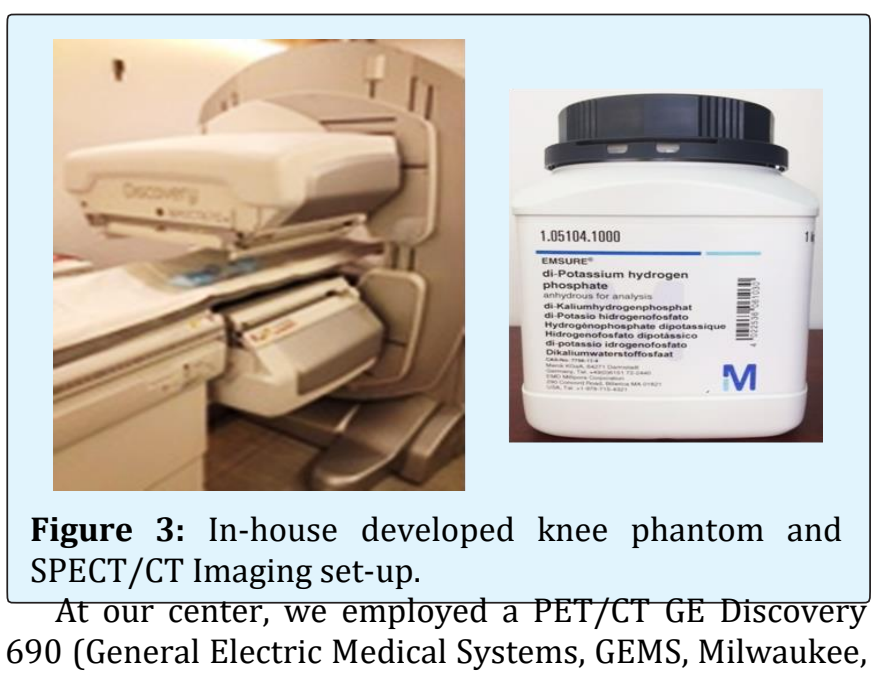


WI, USA) with Cerium activated LYSO crystals and a 64 slice CT for anatomical and attenuation correction. ${ }^{90} \mathrm{Y}$ PET was acquired using positron fraction $3.186 \times 10-5$, half-life $64.1 \mathrm{~h}, 15 \mathrm{~min}$ per bed position and maximum axial field-of-view (AFOV) of $15.7 \mathrm{~cm}$ (Figure 4).

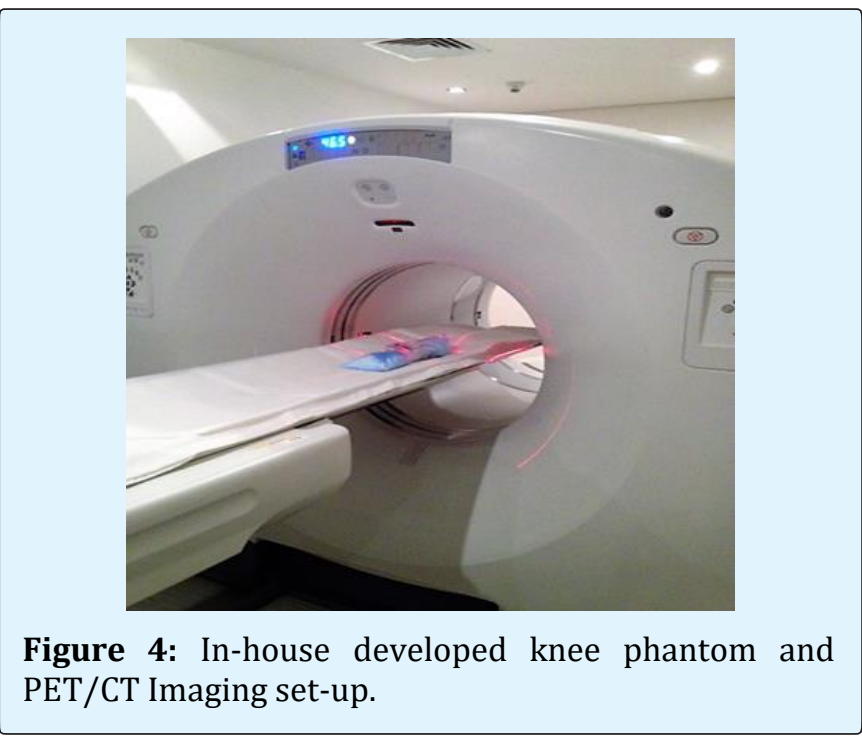

${ }^{90} \mathrm{Y}$ bremsstrahlung SPECT/CT was performed on a GE670 discovery, which has a dual head gamma camera and 16 slice CT. A medium energy general purpose collimator was used based on our earlier literature review. The energy windows were set at lower and upper limit of $55 \mathrm{KeV}$ and $285 \mathrm{keV}$ to capture the most prominent photopeak amidst a continuous bremsstrahlung spectrum (Figure 5).

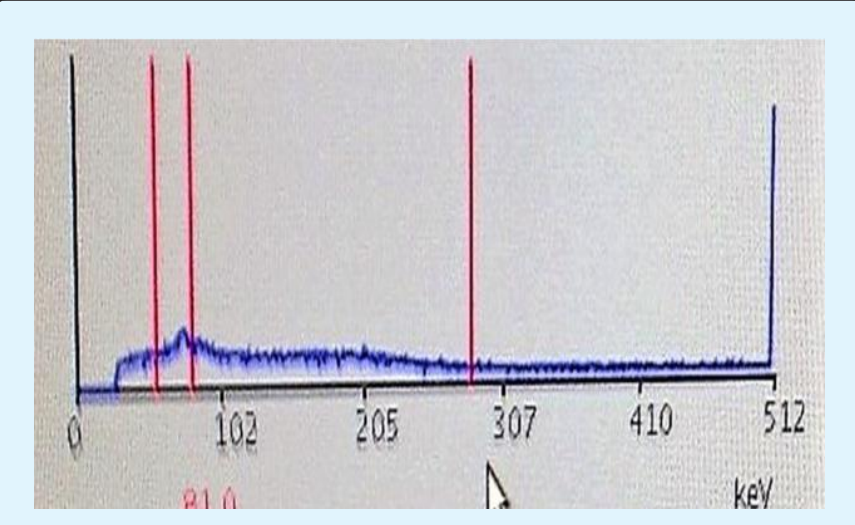

Figure 5: Experimental bremsstrahlung spectrum and energy window set-up for 90Y SPECT scan.

Scans were acquired in 60 frames over $360^{\circ}, 25 \mathrm{~s}$ per frame; Matrix size $128 \times 128$ and low-dose 16 slice CT for anatomical correlation and attenuation correction. All paired ${ }^{90} \mathrm{Y}$ PET/CT and ${ }^{90} \mathrm{Y}$ bremsstrahlung SPECT/CT scans were performed within $30 \mathrm{~min}$ of each other. The acquired data and the reconstructed images were assessed for image resolution, the ability to confirm technical success and detection of non- target activity. Imaging tests were performed on 4 separate occasions with the same amount of activity at each time. Acquisition and processing parameters were kept unchanged to maintain constancy.

\section{Results}

For PET image acquisition and reconstruction, we used an Algorithm which incorporates time of flight and point spread function information and non-time of flight (GEMS name: VUE point FX; Sharp IR; VUE.HD) together with fully three dimensional (3D) ordered subset expectation maximization (3DOSEM). The acquired data were reconstructed with $3 \& 5$ iterations and $18 \& 32$ subsets. The resulting images were corrected for attenuation, scatter, random, dead time, and normalization. (Figures 6, 8 \& 9) SPECT images were reconstructed utilizing 3D-OSEM with 3 iterations and 18 subsets and corrected for anatomical correlation and attenuation correction using a Low-dose 16 slice CT was. The acquired data and the reconstructed images were assessed for image resolution, the ability to confirm technical success and detection of non- target activity. However, the ${ }^{90} \mathrm{Y}$ activity was embedded in a standard vial and as such we did not investigate the detection of nontarget activity at this stage.

${ }^{90} \mathrm{Y}$ PET images appeared to be suffering from low true coincident rate. The authors considered increasing scan time (from $15 \mathrm{~min}$ to $30 \mathrm{~min}$ ) to compensate for low true coincidence rates in an effort to improve image quality. Qualitative evaluation based on visual inspection of phantom images by an expert demonstrated superior resolution of ${ }^{90} \mathrm{Y}$ PET/CT over bremsstrahlung SPECT with a clear edge definition of the phantom $(5 \mathrm{~cm} \mathrm{H} \mathrm{x} 1 \mathrm{~cm}$ D containing less than $1 \mathrm{ml}{ }^{90} \mathrm{Y}$ ) (Figures $7 \& 8$ ).

Following the success of the preliminary study, the authors have instigated a plan to perform further qualitative and quantitative imaging and analysis in relation to target and non-target ROIs, utilising subcentimetre active ROIs. The authors are aiming to employ the modified procedure on patient imaging in conjunction with SPECT. It is anticipated that the PET scan of patients for microsphere bio-distribution in radiosynovectomy will be the first time to be carried out in the state of Kuwait. 

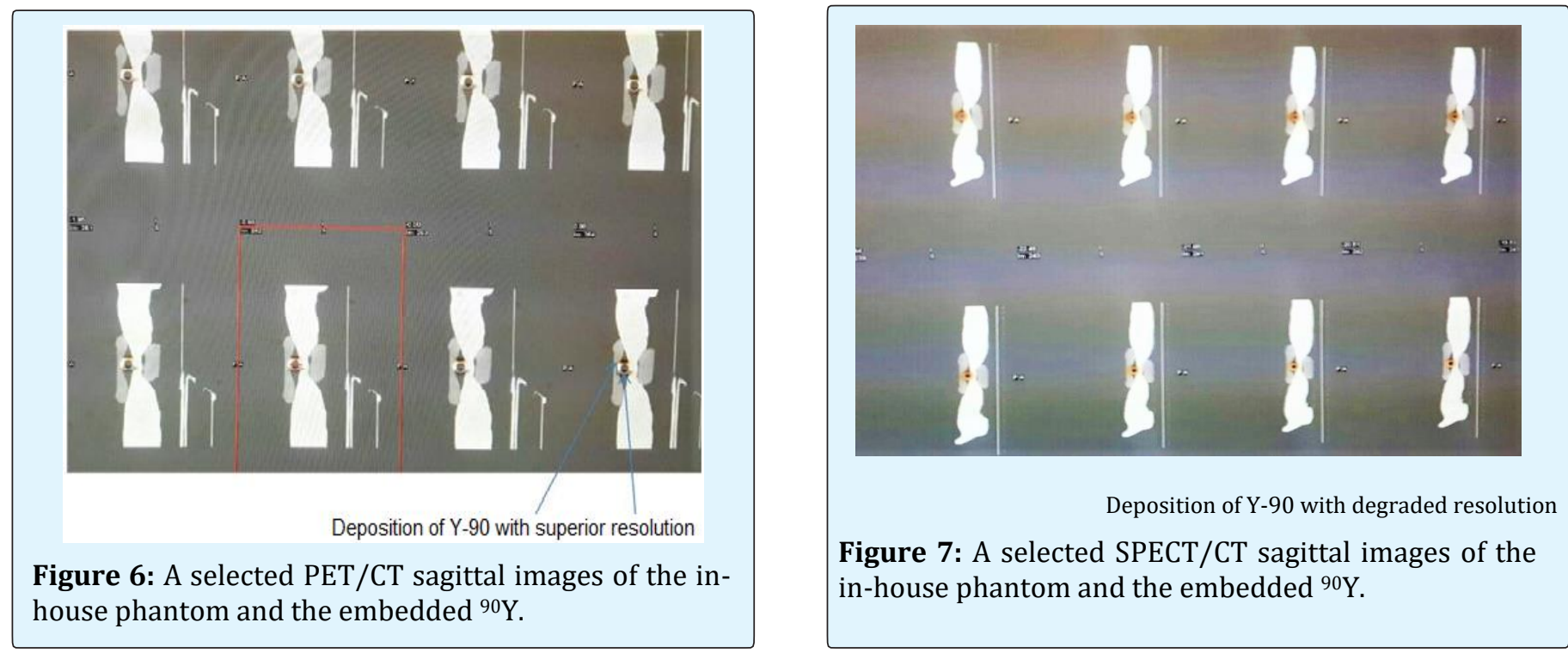

Figure 7: A selected SPECT/CT sagittal images of the in-house phantom and the embedded ${ }^{90} \mathrm{Y}$.

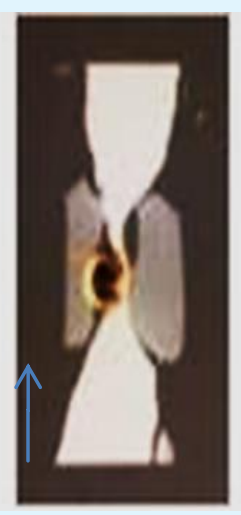

(a)

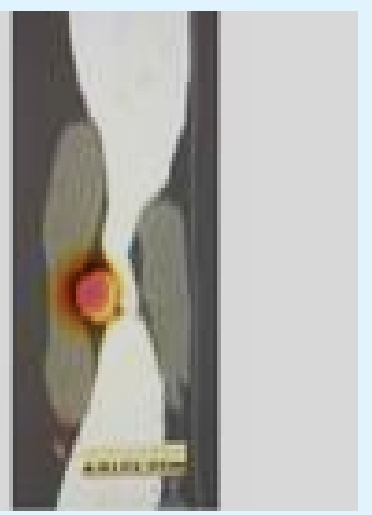

(b)

Figure 8: An enlarged PET/CT (a) and SPECT/CT fussed (b) sagittal images of the knee phantom for visual and edge definition.

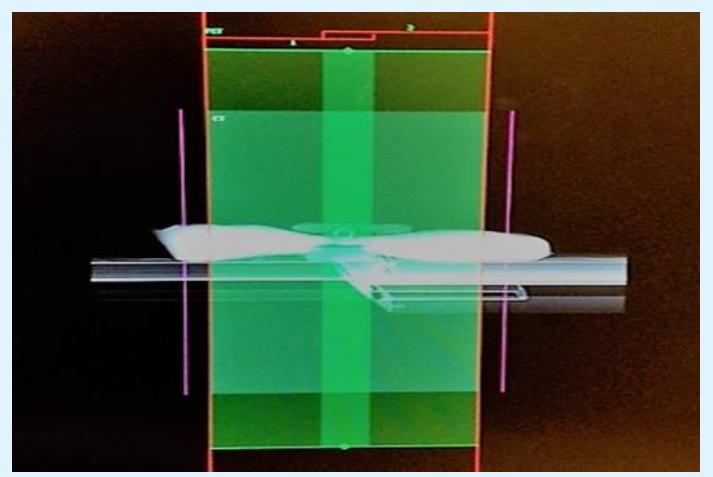

Figure 9: CT set up and imaging: for attenuation correction and co - registration of PET Images.

\section{Conclusion}

The preliminary study using a developed in-house phantom to imitate the knee region is suggesting, although ${ }^{90} \mathrm{Y}$ PET is suffering from low true counts, but it has demonstrated qualitative superiority. The result is suggesting that ${ }^{90} \mathrm{Y}$ PET-CT imaging can serve in two primary clinical roles in radiosynovectomy: (1) evaluating technical success (2) predicting treatment efficacy. It is further suggesting that conclusive information about quantitative and qualitative assessment of ${ }^{90} \mathrm{Y}$ radionuclide imaging can be obtained and it is feasible to establish a platform for the follow up in-vivo (patient study) imaging utilizing the state of art TOF PET/CT at the molecular level. Furthermore, the information obtained 
can also be used to provide patient specific treatment planning for successive therapies and potentially improving response and therefore the quality patient care. The authors are aiming on the next phase to implement the technique on number of patient cases as the initial results are promising.

\section{Acknowledgement}

The Authors and the associated collaborative institutions would like to thank Kuwait Foundation for the Advancement of Sciences (KFAS) for its continuous financial support via the research grant (KFAS - P115$93 \mathrm{MN}-02$ ) which facilitated the initial phase of this investigation utilizing the state of art technology, technique and advanced science.

\section{References}

1. Thomas W Barber, Kenneth SK Yap, Victor Kalff (2012) PET/CT imaging of 90Y radiation synovectomy. EJNMMI 39(5): 917-918.

2. Shen S, DeNardo GL, Yuan A, DeNardo DA, DeNardo SJ (1994) Planar gamma camera imaging and quantitation of yttrium 90 bremsstrahlung. J Nucl Med 35(8): 1381138-1381139.

3. Rault E, Clementel E, Vandenberghe S, D'Asseler Y, Van Holen R, et al. (2010) Comparison of yttrium 90 SPECT and PET mages. J Nucl Med 51(suppl): 125.

4. Elschot M, Nijsen JF, Dam AJ, de Jong HW (2011) Quantitative evaluation of scintillation camera imaging characteristics of isotopes used in liver radioembolization. PLoS One 6: e26174.

5. Minarik D, Sjögreen Gleisner K, Ljungberg M (2008) Evaluation of quantitative (90)Y SPECT based on experimental phantom studies. Phys Med Biol 53(20): 5689-5703.

6. Fabbri C, Sarti G, Cremonesi M, Ferrari M, Di Dia A, et al. (2009) Quantitative analysis of 90Y Bremsstrahlung SPECTCT images for application to 3D patient specific dosimetry. Cancer Biother Radiopharm 24(1): 145-154.

7. Ito S, Kurosawa H, Kasahara H, Teraoka S, Ariga E, et al. (2009) (90)Y bremsstrahlung emission computed tomography using gamma cameras. Ann Nucl Med 23(3): 257-267.
8. Selwyn RG, Nickles RJ, Thomadsen BR, DeWerd LA, Micka JA (2007) A new internal pair production branching ratio of $90 \mathrm{Y}$ : the development of a nondestructive assay for $90 \mathrm{Y}$ and $90 \mathrm{Sr}$. Appl RadiatIsot 65(3): 318-327.

9. Lhommel R, van Elmbt L, Goffette P, Van den Eynde M, Jamar F, et al. (2010) Feasibility of 90Y TOF PETbaseddosimetry in liver metastasis therapy using SIR spheres. EJNMMI 37(9): 1654-1662.

10. Van Elmbt L, Vandenberghe S, Walrand S, Pauwels S, Jamar F (2011) Comparison of yttrium 90 quantitative imaging by TOF and nonTOF PET in a phantom of liver selective internal radiotherapy. Phys Med Biol 56(21): 6759-6777.

11. Walrand S, Flux GD, Konijnenberg MW, Valkema R, Krenning EP, et al. (2011) Dosimetry of yttrium labelled radiopharmaceuticals for internal therapy: $86 \mathrm{Y}$ or $90 \mathrm{Y}$ imaging? Eur J Nucl Med Mol Imaging 38(suppl): 57-68.

12. Bettinardi V, Presotto L, Rapisarda E, Picchio M, Gianolli L, et al. (2011) Physical performance of the new hybrid PET/CT Discovery690. Med Phys 38(10): 5394-5411.

13. Bendriem B, Soussaline F, Campagnolo R, Verrey B, Wajnberg P, et al. (1986) A technique for the correction of scattered radiation in a PET system using time of flight information. J Comput Assist Tomogr 10(2): 287-295.

14. Werner ME, Surti S, Karp JS (2006) Implementation and evaluation of a 3D PET single scatter simulation with TOF modeling. Proc IEEE Nuclear Science SympConf Rec 3: 1768-1773.

15. Watson CC (2007) Extension of single scatter simulation to scatter correction of time of flight PET. IEEE Trans Nucl Sci 54: 1679-1686.

16. Lhommel R, Goffette P, Van den Eynde M, Jamar F, Pauwels S, et al. (2009) Yttrium90 TOF PET scan demonstrates the high resolution distribution after liver SIRT. EJNMMI 36(10): 1696.

17. Kao YH, Tan EH, Ng CE, Goh SW (2011) Yttrium 90 time of flight PET/CT is superior to Bremsstrahlung SPECT/CT for post radioembolization imaging of microsphere biodistribution. ClinNucl Med 36(12): 186-187. 


\section{International Journal of Nuclear Medicine \& Radioactive Substances}

18. Wissmeyer M, Heinzer S, Majno P, Buchegger F, Zaidi $\mathrm{H}$, et al. (2011) 90Y Timeofflight PET/MR on a hybrid scanner following liver radioembolisation (SIRT). EJNMMI 38(9): 1744-1745.

19. Carlier T, Eugène T, Bodet Milin C, Garin E, Ansquer C, et al. (2013) Assessment of acquisition protocols for routine imaging of Y90 using PET/CT. EJNMMI Res 3(1): 11.
20. Willowson K, Forwood N, Jakoby BW, Smith AM, Bailey DL (2012) Quantitative (90)Y image reconstruction in PET. Med Phys 39(11): 7153-7159.

21. Goedicke A, Berker Y, Verburg F, Behrendt F, Winz O, et al. (2013) Studyparameter impact in quantitative 90Yttrium PET imaging for radioembolization treatment monitoring and dosimetry. IEEE Trans Med Imaging 32(3): 485-492. 